Article

\title{
The Three-Month Effects of a Ketogenic Diet on Body Composition, Blood Parameters, and Performance Metrics in CrossFit Trainees: A Pilot Study
}

\author{
Wesley C. Kephart ${ }^{1,2,+}$, Coree D. Pledge ${ }^{3}$, Paul A. Roberson ${ }^{1}$, Petey W. Mumford ${ }^{1}$, \\ Matthew A. Romero ${ }^{1}$, Christopher B. Mobley ${ }^{1}$, Jeffrey S. Martin ${ }^{1,4}{ }^{(D)}$, Kaelin C. Young ${ }^{1,4}$, \\ Ryan P. Lowery ${ }^{5}$, Jacob M. Wilson ${ }^{5}$, Kevin W. Huggins ${ }^{3}$ and Michael D. Roberts ${ }^{1,4, *, t}$ \\ 1 School of Kinesiology, Auburn University, Auburn, AL 36849, USA; kephartw@uww.edu (W.C.K.); \\ par0021@auburn.edu (P.A.R.); pwm0009@auburn.edu (P.W.M.); mzr0049@auburn.edu (M.A.R.); \\ moblecb@auburn.edu (C.B.M.); jmartin@auburn.vcom.edu (J.S.M.); kyoung@auburn.vcom.edu (K.C.Y.) \\ 2 Department of Health, Physical Education, Recreation and Coaching, University of Wisconsin-Whitewater, \\ Whitewater, WI 53190, USA \\ 3 Department of Nutrition, Dietetics and Hospitality Management, Auburn University, \\ Auburn, AL 36849, USA; cdp0017@auburn.edu (C.D.P.); huggikw@auburn.edu (K.W.H.) \\ 4 Department of Cell Biology and Physiology, Edward Via College of Osteopathic Medicine, \\ Auburn, AL 36830, USA \\ 5 Applied Sports Performance Institute, Tampa, FL 33607, USA; rlowery@theaspi.com (R.P.L.); \\ jwilson@theaspi.com (J.M.W.) \\ * Correspondence: mdr0024@auburn.edu; Tel.: +1-334-844-1925; Fax: +1-334-944-1467 \\ + Both authors equally contributed to this work.
}

Received: 21 December 2017; Accepted: 4 January 2018; Published: 9 January 2018

\begin{abstract}
Adopting low carbohydrate, ketogenic diets remains a controversial issue for individuals who resistance train given that this form of dieting has been speculated to reduce skeletal muscle glycogen levels and stifle muscle anabolism. We sought to characterize the effects of a 12-week ketogenic diet (KD) on body composition, metabolic, and performance parameters in participants who trained recreationally at a local CrossFit facility. Twelve participants (nine males and three females, $31 \pm 2$ years of age, $80.3 \pm 5.1 \mathrm{~kg}$ body mass, $22.9 \pm 2.3 \%$ body fat, 1.37 back squat: body mass ratio) were divided into a control group (CTL; $n=5)$ and a KD group $(n=7)$. KD participants were given dietary guidelines to follow over 12 weeks while CTL participants were instructed to continue their normal diet throughout the study, and all participants continued their CrossFit training routine for 12 weeks. Pre, 2.5-week, and 12-week anaerobic performance tests were conducted, and pre- and 12-week tests were performed for body composition using dual X-ray absorptiometry (DXA) and ultrasound, resting energy expenditure (REE), blood-serum health markers, and aerobic capacity. Additionally, blood beta hydroxybutyrate (BHB) levels were measured weekly. Blood BHB levels were 2.8- to 9.5-fold higher in KD versus CTL throughout confirming a state of nutritional ketosis. DXA fat mass decreased by $12.4 \%$ in KD $(p=0.053)$. DXA total lean body mass changes were not different between groups, although DXA dual-leg lean mass decreased in the KD group by $1.4 \%(p=0.068)$, and vastus lateralis thickness values decreased in the KD group by $\sim 8 \%$ $(p=0.065)$. Changes in fasting glucose, HDL cholesterol, and triglycerides were similar between groups, although LDL cholesterol increased $\sim 35 \%$ in $\mathrm{KD}(p=0.048)$. Between-group changes in REE, one-repetition maximum (1-RM) back squat, $400 \mathrm{~m}$ run times, and $\mathrm{VO}_{2 \text { peak }}$ were similar between groups. While our $n$-sizes were limited, these preliminary data suggest that adopting a ketogenic diet causes marked reductions in whole-body adiposity while not impacting performance measures in recreationally-trained CrossFit trainees. Whether decrements in dual-leg muscle mass and vastus lateralis thickness in KD participants were due to fluid shifts remain unresolved, and increased LDL-C in these individuals warrants further investigation.
\end{abstract}


Keywords: ketogenic diet; body composition; power; strength

\section{Introduction}

Dietary practices that facilitate losses in body fat while maintaining or leading to an accretion of muscle mass have been of interest to individuals who resistance train recreationally. In this regard, there is overwhelming support that higher protein diets are able to improve muscle mass gains while not affecting body fat [1-4]. However, more recent data from our laboratory in rodents [5,6], and other evidence in humans [7], suggest that adopting a low-carbohydrate, moderate-protein, high-fat ketogenic diet (KD) may also serve to support muscle mass gains with resistance training while promoting decreases in adiposity. Notwithstanding, there are still scientific and mainstream commentaries that posit this form of dieting is detrimental to individuals who resistance train. Namely, given that the diet is very low in carbohydrates, it has been posited that a KD will reduce skeletal muscle glycogen levels and chronically increase intramuscular AMP-activated protein kinase (AMPK) signaling. Indeed, these findings have been reported in mice [8,9], and have led the authors to speculate that KD feeding may lead to a chronic suppression of mammalian target of rapamycin complex 1 (mTORc1) signaling and muscle protein synthesis, as well as an eventual stagnation in the anabolic response to resistance training and/or a loss in muscle mass in humans.

To counter these hypotheses, we have demonstrated that six weeks of KD feeding in rats does not impair muscle glycogen levels, reduce mTORc1 signaling markers, or affect basal muscle protein synthesis levels when compared with rats fed an isocaloric Western diet (WD) [5]. Additionally, we observed that KD- and Western diet (WD)-fed rats experienced similar increases in hind limb muscle masses and ribosome biogenesis when voluntarily trained on resistance-loaded running wheels over a six-week period. In humans, Paoli et al. [10] reported that elite male artistic gymnasts adopting a KD for 30 days during a normal training schedule experienced a robust decrease in whole-body adiposity, but no significant impairment of muscular strength or reduction in muscle mass. Wilson et al. [7] recently reported that male bodybuilders adopting a KD for 10 weeks experienced similar increases in muscle mass and strength, as well as similar reductions in fat mass, compared with bodybuilders consuming a mixed macronutrient diet. Gregory et al. [11] reported that six weeks of KD reduced fat mass by $2.8 \mathrm{~kg}$ on average without affecting lean body mass in non-elite CrossFit athletes. Additionally, power metrics, along with time to complete a circuit-training style workout, was not impaired when compared with athletes consuming their typical diet. Hence, these recent data counter molecular evidence indicating that adopting a KD will lead to muscle mass loss or performance decrements in anaerobic athletes. It should be noted, however, that recent data also indicate that shorter-term ketogenic diets could negatively alter physiological adaptations to aerobic training. In this regard, Burke et al. [12] reported that a three-week KD increased the oxygen cost during a two-hour treadmill race-walk in world-class race walkers. Thus, more studies examining the physiological and performance effects of ketogenic diets are warranted.

Herein, we sought to characterize the effects of a 12-week KD on body composition, metabolic, and performance parameters in participants who trained recreationally at a local CrossFit facility. Based on the supporting literature, we hypothesized that participants would experience reductions in whole-body adiposity but would not experience losses in lean body mass or impairments in performance metrics.

\section{Materials and Methods}

\subsection{Ethics Approval and Subject Recruitment and Consent}

Prior to initiating this study, the protocol was reviewed and approved by the Auburn University Institutional Review Board (approved protocol \#: 16-031 MR 1603; IRB contact: irbadmin@auburn.edu) 
and was in compliance with the Helsinki Declaration. Twelve subjects were recruited from a local CrossFit gymnasium in the Auburn community to participate in the study. As inclusion criteria, we established that participants must have: (a) been active members at the gymnasium for at least three months; (b) been between the ages of 19-45; and (c) displayed a strength:mass ratio (determined via one-repetition maximum back squat) of at least 1.00 .

\subsection{Study Design}

A depiction of the study design is presented in Figure 1. Briefly, an initial meeting at the School of Kinesiology at Auburn University between study investigators and participants was held whereby all 12 participants were informed about the purpose of the study and the study design, and educated about the KD. Thereafter, participants were given a choice to participate in the KD group or continue their normal diet (CTL). In an effort to optimize compliance with KD guidelines, participants who verbalized interest with adopting a KD for 12 weeks were placed in the KD group $(n=7)$, while others were placed in the CTL group $(n=5)$. Participants then completed a screening and health history questionnaire to ensure inclusion criteria were met and that there were no risk factors that might be aggravated by maximal free-weight strength testing and aerobic testing (e.g., musculoskeletal injuries or exercise-induced asthma). Participants were then told to maintain their pre-study diet and scheduled for baseline testing which commenced $\sim 1$ week following the initial meeting.

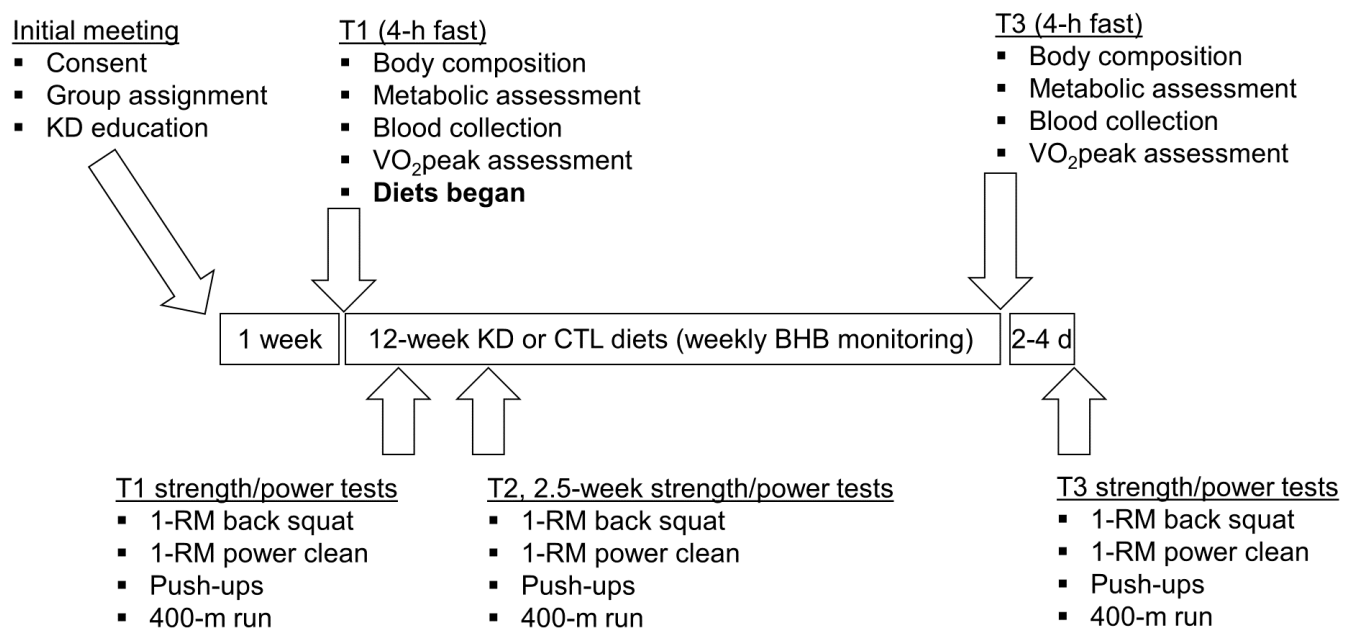

Figure 1. Study design. This figure depicts the 12-week dietary intervention. Notably, all participants continued their normal workout routine at the local CrossFit training facility during the study. Abbreviations: KD, ketogenic diet; CTL, control diet; 1-RM, one repetition maximum.

\subsection{Body Composition, Metabolic, and Aerobic Performance Assessments}

During T1 (pre-test) and T3 (post-test) participants were instructed to arrive to the School of Kinesiology at Auburn University either in the morning or evening in a 4-h fasted state in order to best fit their work schedules; notably T1 and T3 time of day testing was similar for each participant. Upon arrival to the laboratory, participants submitted a urine sample $(\sim 5 \mathrm{~mL})$ to assess for normal hydration specific gravity levels (1.005-1.020 ppm) using a handheld refractometer (ATAGO; Bellevue, WA, USA). Participants with a urine specific gravity $\geq 1.020$ were asked to consume tap water every $15 \mathrm{~min}$ for $30 \mathrm{~min}$ and then were re-tested. Following hydration testing, height (T1 only) and body mass were assessed using a digital column scale (Seca 769; Seca, Chino, CA, USA). Next, participants were subjected to a full body dual X-ray absorptiometry (DXA) scan while wearing general sports attire (i.e., athletic shorts or compression shorts and an athletic shirt) to assess various body composition characteristics. Notably, body segmentation for each scan was standardized prior to analyses by the same technician (Wesley C. Kephart), and total body lean mass and fat mass was 
assessed by the accompanying software. According to previous data published by our laboratory, the same-day reliability of the DXA during a test-calibrate-retest on 10 participants produced intra-class correlation coefficients of 0.998 for total body fat mass (mean difference between tests $=0.40 \pm 0.05 \mathrm{~kg}$ ), and 0.998 for total lean body mass (mean difference between tests $=0.29 \pm 0.13 \mathrm{~kg}$ ) [13].

Following body composition assessments, resting energy expenditure (REE) and respiratory quotient (RQ) assessments were performed in a supine position over a 10-min period in a dark and quite laboratory space. Expired gases during the tests were continuously analyzed over the 10-min period using a TrueMax 2400 metabolic measurement system (ParvoMedics, Sandy, UT, USA) with a dilution pump which was used to set the fraction of end tidal $\mathrm{CO}_{2}\left(\mathrm{FECO}_{2}\right)$ between $0.90-1.10$. All data were averaged in $1 \mathrm{~min}$ intervals, and the average of the last $5 \mathrm{~min}$ of the test was used for REE and RQ calculations.

Following REE assessments, participants donated a venous blood sample (described in greater detail below), and then performed $\mathrm{VO}_{2 \text { peak }}$ testing on a motorized treadmill (Waukesha, Woodway, WI, USA) using a graded protocol. Briefly, participants were allowed to warm-up by walking on the treadmill at $4.8 \mathrm{~km} / \mathrm{h}(3.0 \mathrm{mph})$ for $3 \mathrm{~min}$. After the warm-up was completed, the treadmill incline was set to $1 \%$ and belt speed was set to the speed pre-indicated by the participants as a comfortable $5-\mathrm{km}$ running pace. Thereafter, the treadmill grade increased by $2 \%$ every $2 \mathrm{~min}$ until the participants indicated volitional fatigue. Expired gases during the tests were continuously analyzed using a TrueMax 2400 metabolic measurement system (ParvoMedics, Sandy, UT, USA), averaged in 15-s intervals, and the highest 15-s average for $\mathrm{VO}_{2}$ was denoted as the $\mathrm{VO}_{2 \text { peak }}$.

\subsection{Blood-Serum Collection and Analysis}

Blood was collected in a 4-h fasted state at $\mathrm{T} 1$ and $\mathrm{T} 3$ from the antecubital vein and allowed to clot at room temperature. Thereafter, tubes were centrifuged at $3500 \mathrm{~g}\left(23^{\circ} \mathrm{C}\right)$ for $10 \mathrm{~min}$. Serum was aliquoted in $1.7 \mathrm{~mL}$ microtubes and stored at $-80^{\circ} \mathrm{C}$. Once the study was completed, all tubes were transported to the East Alabama Medical Center (Opelika, AL, USA) whereby blood glucose and lipids were determined. Additionally, whole-blood (2-h post prandial) beta-hydroxbutyrate (BHB) concentrations were determined weekly via finger sticks using a handheld analyzer and testing strips (CardioChek, Indianapolis, IN, USA).

\subsection{Anaerobic Performance Assessments}

Approximately 48-96 h following T1 and T3, as well as two and one-half weeks into the intervention, participants performed one repetition maximum (1-RM) back squat and power clean assessments using a $20 \mathrm{~kg}$ barbell (York Barbell) and free weights at the local CrossFit gymnasium (Backbone CrossFit, Auburn, AL, USA). The demonstration of proper technique as well as the implementation of progressively-loaded RM testing were overseen by Wesley C. Kephart and Coree D. Pledge, who have vast experience with strength and performance testing [14-16]. A repetition was not counted if subjects exhibited poor and/or unsafe technique or needed assistance with a repetition during maximal testing. Approximately $10 \mathrm{~min}$ following 1-RM assessments, maximal repetition pushup tests were performed to volitional fatigue. Finally, 400-m run assessments were performed on an outdoor recreational track ( $400 \mathrm{~m} / \mathrm{lap}$ ) using a handheld timer. Unlike the laboratory tests, where participants were instructed to arrive following a 4-h fast, no mandated fasting was implemented prior to these performance tests.

\subsection{Dietary Guidelines and Nutritional Intake Monitoring}

Following the T1 anaerobic testing procedures, participants in the KD group were given general KD guidelines and handouts of sample meal plans published by Volek and Phinney [17]. Participants in the CTL group were instructed to maintain their normal dietary habits throughout the study. Only KD subjects were asked to complete three-day food logs (two weekdays and one weekend day) to return within one week of T1 and T3 testing. On each occasion, participants were given detailed written and 
verbal instructions on completing the food logs. Dietary intake data were analyzed using open-sourced software (http:/ / www.myfitnesspal.com), which has been employed by our laboratory to analyze macronutrient data [15].

\subsection{Statistical Analyses and Data Presentation}

All data herein are presented in figures as means \pm standard error values with individual subject plots and statistical analyses were performed using SPSS v22.0 (IBM, Armonk, NY, USA). With the exception of weekly blood BHB levels, which were compared between groups using independent samples $t$-tests, all dependent variables were examined between groups using two-way (group $\times$ time) repeated measures analysis of variances (ANOVAs). Huynh-Feldt (H-F) corrections were applied for data where the assumption of sphericity was violated. Given our limited $n$-sizes, partial eta-squared values $\left(\eta_{\mathrm{p}}{ }^{2}\right)$ for group $\times$ time interactions, and interactions were further interrogated if they were significant $(p<0.05)$ or effect sizes were large $\left(\eta_{\mathrm{p}}{ }^{2}>0.14\right)$. In these cases, within-group dependent samples $t$-tests as well as between-group independent samples $t$-tests were performed as post hoc tests, and $p$-values between 0.05 and 0.10 (i.e., 'approaching significance') were considered meaningful due to limited $n$-sizes.

\section{Results}

\subsection{Baseline Characteristics between Treatments}

Baseline characteristics between groups are presented in Table 1. Independent $t$-tests indicated that there were no between-group differences for age, height, body mass, DXA body fat percentage, or strength:mass ratio. Notably, one KD participant presented with a strength: body mass ratio of 0.93 , but the investigators decided to include the subject in the analyses due to limited $n$-sizes.

Table 1. Baseline characteristics between groups.

\begin{tabular}{cccc}
\hline Variable & KD $(\boldsymbol{n}=\mathbf{7})$ & CTL $(\boldsymbol{n}=\mathbf{5})$ & $\boldsymbol{p}$-Value \\
\hline Males /Females $(n)$ & $5 / 2$ & $4 / 1$ & - \\
Age (years) & $32 \pm 3$ & $29 \pm 3$ & 0.592 \\
Height $(\mathrm{m})$ & $1.71 \pm 0.08$ & $1.70 \pm 0.03$ & 0.954 \\
Body mass $(\mathrm{kg})$ & $82.7 \pm 8.2$ & $76.9 \pm 5.5$ & 0.601 \\
DXA body fat $(\%)$ & $24.6 \pm 2.2$ & $20.6 \pm 4.7$ & 0.420 \\
Strength: mass & $1.35 \pm 0.13$ & $1.42 \pm 0.18$ & 0.736 \\
\hline
\end{tabular}

Notes: Data are presented as means \pm SE values. Strength: mass ratio was determined by dividing the one repetition maximum back squat $(\mathrm{kg})$ by baseline body mass $(\mathrm{kg})$. Abbreviations: $\mathrm{KD}$, ketogenic diet group; $\mathrm{CTL}$, control diet group; $n$, number of participants; $m$, meters; $k g$, kilograms.

\subsection{Self-Reported Macronutrient Intakes and Workouts Completed over the Intervention}

As noted previously, only the KD participants completed food logs. Of the seven KD participants, only four returned the $\mathrm{T} 3$ food logs. Notably, self-reported carbohydrate consumption decreased $(\mathrm{T} 1=164 \pm 32 \mathrm{~g} / \mathrm{d}, \mathrm{T} 3=15 \pm 3 \mathrm{~g} / \mathrm{d}, p=0.014)$ and total calorie consumption decreased $(\mathrm{T} 1=2499 \pm 350 \mathrm{kcal} / \mathrm{d}, \mathrm{T} 3=1948 \pm 293 \mathrm{kcal} / \mathrm{d}, p=0.032)$. While self-reported dietary fat values were greater $(\mathrm{T} 1=154 \pm 40 \mathrm{~g} / \mathrm{d}, \mathrm{T} 3=170 \pm 25 \mathrm{~g} / \mathrm{d}, p=0.576)$ and protein values were lower $(\mathrm{T} 1=114 \pm 10 \mathrm{~g} / \mathrm{d}, \mathrm{T} 3=89 \pm 20 \mathrm{kcal} / \mathrm{d}, p=0.112)$, there were no significant differences between preand post-values. Additionally, while three participants failed to return post-study food logs, weeks $1-12$ average blood ketone levels for these participants were $0.87,0.95$, and $1.43 \mu \mathrm{M}$, suggesting that they complied with the KD guidelines throughout the study.

Regarding CrossFit workouts completed over the intervention, the KD group completed an average of $27 \pm 3$ workouts, whereas the CTL group completed an average of $20 \pm 5$ workouts $(p=0.245$ between conditions). 


\subsection{Weekly Blood Ketone Levels and Changes in Body Composition Metrics}

Blood BHB levels were 2.8-9.5-fold higher in KD versus CTL throughout the intervention confirming a state of nutritional ketosis in the KD group. In addition, independent samples $t$-tests indicated that humoral BHB levels were significantly greater $(p<0.05)$ in the KD group at weeks 1-6, 9 and 12 (Figure 2a). A main time effect (T1 > T3; $p=0.020$ ) and large effect for the group $\times$ time interaction was observed for change in body mass between groups $(p=0.053$, $\eta_{\mathrm{p}}{ }^{2}=0.325$; Figure $2 \mathrm{~b}$ ); notably a significant decrease from T1 within the KD group was observed $(p=0.022)$. Additionally, a main time effect approached significance (T1 > T3; $p=0.051)$, and a large effect for the group $\times$ time interaction was observed for change in DXA fat mass between groups $\left(p=0.126, \eta_{\mathrm{p}}{ }^{2}=0.218\right.$; Figure $\left.2 \mathrm{c}\right)$; notably, the decrease from T1 within the KD group approached significance $(p=0.053)$. There was no main time effect $(p=0.521)$ or group $\times$ time interaction observed for DXA lean body mass $\left(p=0.534, \eta_{p}{ }^{2}=0.040\right.$; Figure $\left.2 d\right)$. There was no main time effect $(p=0.876)$ or group $\times$ time interaction observed dual-arm DXA lean mass $\left(p=0.742, \eta_{\mathrm{p}}{ }^{2}=0.011\right.$; Figure 2e). There was no main time effect for dual-leg DXA lean mass $(p=0.344)$, although a large effect for the group $\times$ time interaction was observed $\left(p=0.111, \eta_{p}{ }^{2}=0.234\right.$; Figure $\left.2 \mathrm{f}\right)$ whereby lower T3 values in the KD group approached significance $(p=0.068)$. Likewise, there was no main time effect for ultrasound derived vastus lateralis thickness $(p=0.159)$, although a large effect for the group $\times$ time interaction was observed $\left(p=0.180, \eta_{\mathrm{p}}{ }^{2}=0.172\right.$; Figure 2e) whereby lower T3 values in the KD group approached significance $(p=0.065)$.

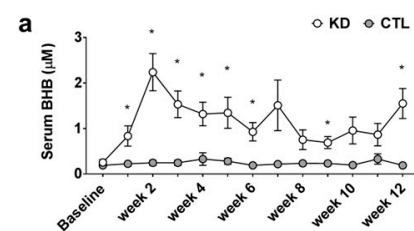

b
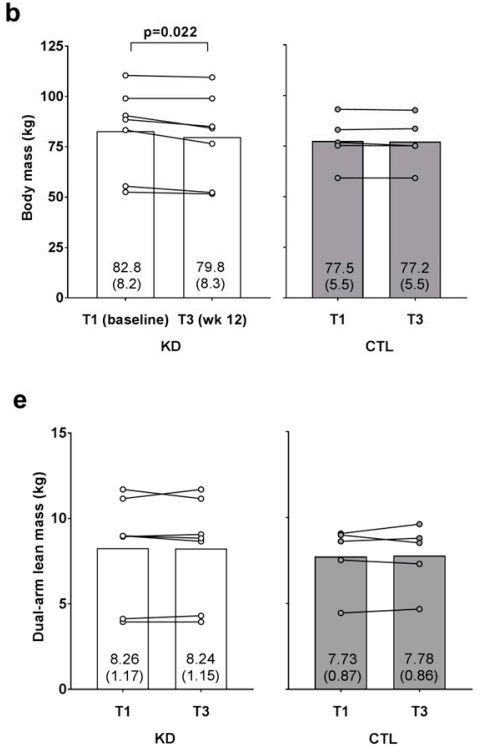

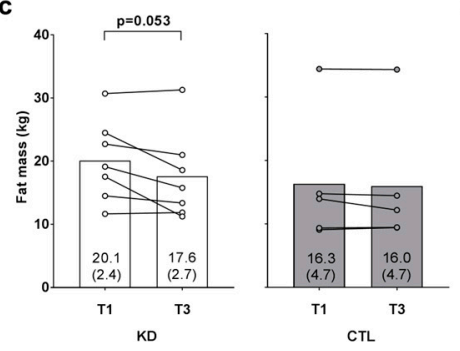

f

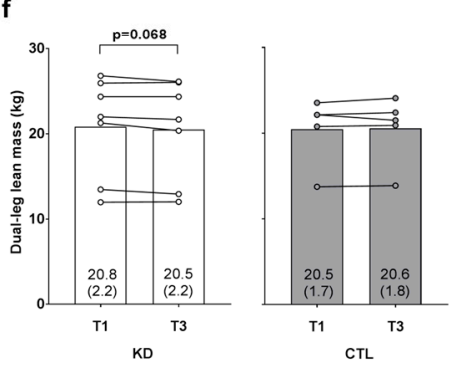

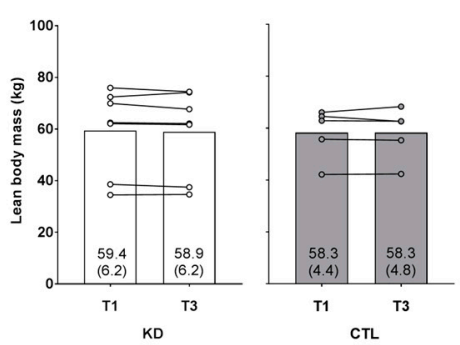

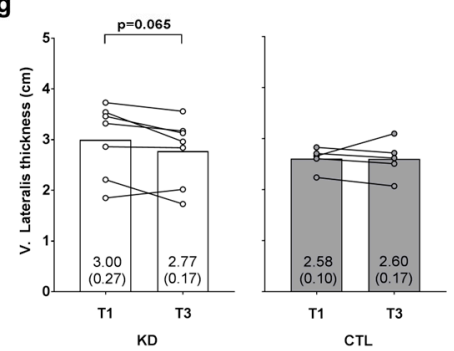

Figure 2. Weekly blood ketone and body composition effects prior to and 12 weeks following the study. Weekly blood beta-hydroxybutyrate (BHB) levels are presented in panel (a); DXA body mass, DXA fat mass, DXA lean body mass, DXA dual-arm lean mass, DXA dual-leg lean mass, and vastus lateralis thickness (assessed via ultrasound) are presented in panels (b-g); Data in panel (a) are presented as means \pm SE; Bar graph data in panels $(\mathbf{b}-\mathbf{g})$ are presented as group means accompanied by individual participant values, and means $( \pm S E)$ values are indicated the bottom portion of each bar. Abbreviations: KD, ketogenic diet; CTL, control diet; T1, baseline testing; T3, 12-week post-testing. Symbols: * indicates between-group difference in blood $\mathrm{BHB}(p<0.05)$. 


\subsection{Changes in Resting Metabolic Metrics}

There was no time $(p=0.781)$ or group $\times$ time interaction observed for resting energy expenditure $\left(p=0.613, \eta_{\mathrm{p}}^{2}=0.024\right.$; Figure 3a). There was a significant time effect $(\mathrm{T} 1>\mathrm{T} 3, p=0.002)$ observed for resting $R Q$, although there was no group $\times$ time interaction $\left(p=0.916, \eta_{\mathrm{p}}^{2}=0.001\right.$; Figure $\left.3 b\right)$.
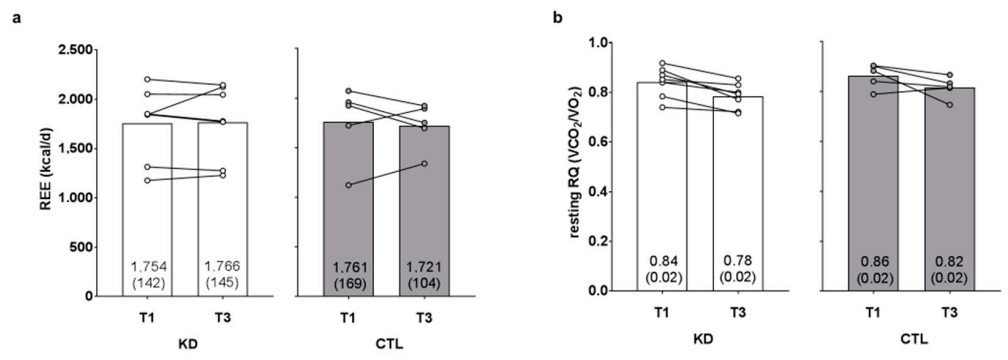

Figure 3. Effects of the intervention on resting energy expenditure and respiratory quotient values. Resting energy expenditure (REE) and resting respiratory quotient (RQ) values are presented in panels $(\mathbf{a}, \mathbf{b})$. Data are presented as group means accompanied by individual participant values, and means $( \pm \mathrm{SE})$ values are indicated at the bottom portion of each bar. Abbreviations: KD, ketogenic diet; CTL, control diet; T1, baseline testing; T3, 12-week post-testing.

\subsection{Changes in Performance Metrics}

There was no main time effect [Huynh-Feldt $(\mathrm{H}-\mathrm{F}) p=0.626]$ or group $\times$ time interaction observed for 1-RM squat (H-F $p=0.422, \eta_{p}^{2}=0.073$; Figure $\left.4 a\right)$. There was no main time effect $(p=0.995$ ) or group $\times$ time interaction observed for 1-RM power clean $\left(p=0.347, \eta_{\mathrm{p}}{ }^{2}=0.100\right.$; Figure $\left.4 \mathrm{~b}\right)$.

There was a main time effect regarding maximum pushup repetitions (T3 $>\mathrm{T} 1$ and $\mathrm{T} 2, p=0.032$ and $p=0.016$, respectively), but no group $\times$ time interaction was observed $\left(\mathrm{H}-\mathrm{F} p=0.716, \eta_{\mathrm{p}}{ }^{2}=0.020\right.$; Figure $4 c)$. There was no main time effect $(p=0.326)$ or group $\times$ time interaction observed for $400-\mathrm{m}$ run time $\left(p=0.479, \eta_{\mathrm{p}}{ }^{2}=0.071\right.$; Figure $\left.4 \mathrm{~d}\right)$. There was no main time effect observed for observed for $\mathrm{VO}_{2 \text { peak }}$ $(p=0.105)$, although a large effect for the group $\times$ time interaction was observed $\left(p=0.188, \eta_{\mathrm{p}}{ }^{2}=0.166\right.$; Figure 4e). However, numerically greater T3 values in the $\operatorname{KD}(p=0.782)$ and CTL $(p=0.109)$ groups did not approach statistical significance.
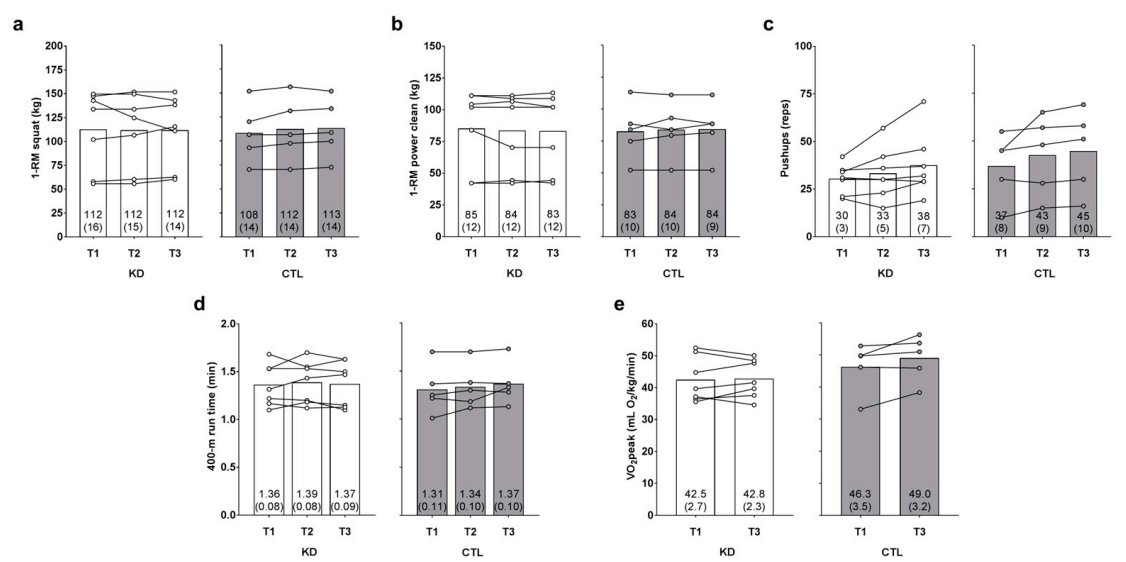

Figure 4. Effects of the intervention on strength, anaerobic, and aerobic performance metrics. One-repetition maximum (1-RM) squat, 1-RM power clean, maximum pushup repetitions, 400-m run time, and $\mathrm{VO}_{2 \text { peak }}$ values (assessed with a graded treadmill test and indirect calorimetry) are presented in panels $(\mathbf{a}-\mathbf{e})$. Data are presented as group means accompanied by individual participant values, and means $( \pm S E)$ values are indicated at the bottom portion of each bar. Abbreviations: KD, ketogenic diet; CTL, control diet; T1, baseline testing; T2, 2.5-week testing; T3, 12-week post-testing. 


\subsection{Changes in Blood-Serum Parameters}

Changes in blood parameters between groups are presented in Table 2. There was no main time effect $(p=0.190)$ or group $\times$ time interaction observed for serum glucose $\left(p=0.881, \eta_{\mathrm{p}}^{2}=0.002\right)$. There was no main time effect $(p=0.409)$ or group $\times$ time interaction observed for HDL cholesterol $\left(p=0.649, \eta_{\mathrm{p}}{ }^{2}=0.022\right)$. There was no main time effect $(p=0.977)$ or group $\times$ time interaction observed for triglycerides $\left(p=0.585, \eta_{\mathrm{p}}{ }^{2}=0.031\right)$. There was no main time effect $(p=0.147)$, although there was a significant and large effect for the group $\times$ time interaction observed for serum LDL cholesterol levels $\left(p=0.029, \eta_{\mathrm{p}}{ }^{2}=0.393\right)$, and post hoc tests indicated within-group increases for the KD group $(p=0.048)$ and significant decreases in the CTL group $(p=0.006)$.

Table 2. Changes in serum parameters between groups.

\begin{tabular}{|c|c|c|c|}
\hline Variable & $\mathrm{KD}(n=7)$ & CTL $(n=5)$ & $\begin{array}{l}\quad \text { Time } p \text {-Value } \\
\text { Group } \times \text { Time } p \text {-Value }\left(\eta_{p}{ }^{2}\right)\end{array}$ \\
\hline \multicolumn{4}{|c|}{ Glucose (mg/dL) } \\
\hline Pre & $84.4 \pm 4.3$ & $79.2 \pm 5.4$ & 0.190 \\
\hline Post & $76.7 \pm 5.5$ & $73.0 \pm 7.4$ & $0.881(0.002)$ \\
\hline \multicolumn{4}{|c|}{ HDL-C (mg/dL) } \\
\hline Pre & $52.3 \pm 3.2$ & $56.6 \pm 2.2$ & 0.409 \\
\hline Post & $51.3 \pm 3.2$ & $53.2 \pm 3.2$ & $0.649(0.022)$ \\
\hline \multicolumn{4}{|c|}{ LDL-C (mg/dL) } \\
\hline Pre & $114.1 \pm 16.2$ & $88.4 \pm 13.9$ & 0.147 \\
\hline Post & $153.9 \pm 27.4^{*},+$ & $79.0 \pm 13.9^{\#}$ & $0.029(0.393)$ \\
\hline \multicolumn{4}{|c|}{ Triglycerides (mg/dL) } \\
\hline Pre & $72.7 \pm 9.5$ & $56.8 \pm 21.3$ & 0.977 \\
\hline Post & $65.9 \pm 7.1$ & $64.4 \pm 20.7$ & $0.585(0.031)$ \\
\hline
\end{tabular}

Notes: Data are presented as means \pm SE values. Abbreviations: KD, ketogenic diet group; CTL, control diet group; $n$, number of participants; HDL-C, high density lipoprotein cholesterol; LDL-C, low density lipoprotein cholesterol. Symbols: ${ }^{*}$ increase within group from T1 to T3 $(p<0.05)$; \# decrease within group from T1 to T3 $(p<0.05) ;{ }^{\dagger}$ higher values approached significance in KD at T3 compared with CTL $(p=0.057)$.

\section{Discussion}

This is one of the first reports to examine the longer-term physiological and performance effects of a ketogenic diet in recreationally resistance-trained individuals. As mentioned previously, we have demonstrated in rats that six weeks of KD feeding does not adversely affect markers of muscle hypertrophy, and that KD- and WD-fed rats experienced similar increases in hind limb muscle masses and molecular markers suggestive of anabolism when voluntarily trained on resistance-loaded running wheels over a six-week period [5]. Likewise, Paoli et al. [10], Wilson et al. [7] and Gregory et al. [11] reported that humans engaging in a KD do not experience anaerobic performance deficits over a 4-10-week period. Herein, we add to these data to suggest that individuals who train recreationally at a CrossFit gym while adopting a KD for 12 weeks experience a reduction in whole-body adiposity with little influence on metabolic or exercise performance measures. It is notable, however, that KD participants did experience decrements in vastus lateralis thickness and dual-leg DXA lean mass following the intervention. These findings counter the Paoli et al. [10], Wilson et al. [7], and Gregory et al. [11] data, although several studies have reported that engaging in a 12-week KD decreases DXA lean body mass in individuals who participated in a diet-only intervention [18-20]. Notably, our body composition findings could be indicative of resultant fluid shifts that occur via KD. While we did confirm a state of adequate hydration via urinalysis, others have reported that four months of KD elicited a marked reduction in DXA lean body mass primarily due to changes in total body water (assessed via bioelectrical impedance) [21]. Given these discordant data, more human studies are needed for clarity regarding shorter-term and longer-term KD effects on whole-body 
hydration, protein metabolism, muscle protein synthesis levels, and muscle fiber histological attributes (i.e., muscle fiber cross sectional area) in exercise-trained individuals.

While no performance decrements were evident in KD participants following the intervention, it is notable that these participants did not experience improvements in certain performance measures relative to CTL participants (e.g., 1-RM squat and $\mathrm{VO}_{2 \text { peak }}$ ). Alternatively stated, the CTL group may have experienced significant improvements in select performance measures had $n$-sizes been larger or the study had been longer in duration. Select commentaries have asserted that individuals who aim to optimize anaerobic endeavors benefit from higher amounts of dietary carbohydrates; case in point, a recent commentary suggests that consuming $2.5 \mathrm{~g} / \mathrm{kg} 3 \mathrm{~h}$ prior to exercise and $9 \mathrm{~g} / \mathrm{kg}$ during post-exercise recovery will benefit stop-and-go athletes [22]. While individuals in the current study were recreationally trained, we observed no performance decrements 2.5 weeks or 12 weeks after engaging in a KD. Indeed, our data agree with others who reported that KD does not adversely affect anaerobic performance $[7,10]$. Moreover, others have reported that aerobic-based athletes experience increases in fat oxidation during running [23], improvements in $\mathrm{O}_{2}$ uptake at lactate threshold after longer-term KD [24], and even improvements in sprint performance [25]. Hence, we contend that the human body is capable of adapting to several different diets during periods of exercise training, and performance may not be compromised so long as caloric needs are met. Notwithstanding, given that KD-induced anaerobic and aerobic performance improvements were not evident herein or in other published reports, we contend that practitioners should explore implementing this diet when body composition improvements are sought rather than performance benefits.

Another interesting finding was that those in the KD group experienced increases in serum LDL cholesterol levels. Human reports are mixed with regard to how partaking in a KD affects serum LDL cholesterol levels, with some data suggesting decreases [18], no effects [20], or increases [19]. Additionally, our findings are limited in that we did not quantify LDL particle size. In this regard, Volek et al. reported that a 12-week KD intervention positively affected LDL particle size, saturated fat content in triglycerides, and Apo B/Apo A-1 ratios [18]. However, it is notable that the subjects in all of these aforementioned studies examined overweight and/or hyperlipidemic individuals. Thus, it remains plausible that otherwise healthy individuals may present increases in LDL cholesterol levels when adopting a KD. To this end, more research is needed to firmly assess how partaking in KD affects blood lipid levels in healthy versus unhealthy individuals.

\section{Limitations and Conclusions}

One obvious limitation to the current study is the limited number of participants. Hence, we feel that it is important to interpret our current data within this context. Additionally, there is the real chance that individuals in the KD group may have experienced a placebo effect in relation to performance outcomes. Alternatively stated, given that many of the KD participants experienced improvements in body composition and (anecdotally) many of these participants perceived themselves having more energy throughout the day, these phenomena could have motivated them to perform the exercise tests with more vigor relative to the CTL group.

To summarize, our data suggest that a 12 -week KD in recreationally-trained CrossFit trainees facilitates improvements in whole-body adiposity without compromising weightlifting, running, or aerobic performance metrics. However, our finding that KD may reduce leg muscle mass suggests that, over prolonged periods, the diet could adversely affect muscle anabolism and warrants further human investigations using sensitive histological techniques. Additionally, given our observation that KD increased serum LDL cholesterol levels, more research is needed regarding how longer-term KD engagement affects blood lipid levels in normolipidemic athletes. Notwithstanding, the novelty of this study examining how a longer term KD affects anaerobic performance metrics continues to contribute to field of sports nutrition and challenges the paradigm regarding very high carbohydrate needs in anaerobic trainees or athletes. 
Acknowledgments: The authors would like to thank all of the participants who devoted time and effort to the study. The authors would also like to thank Mike and Lauren Jones at the Backbone CrossFit gymnasium (Auburn, AL, USA) for allowing our research team to conduct this study at their facility.

Author Contributions: Michael D. Roberts has made substantial contributions to the conception and design, acquisition of data, and analysis and interpretation of data. Wesley C. Kephart, Paul A. Roberson, Coree D. Pledge, Petey W. Mumford, and Michael D. Roberts primarily were involved in drafting the manuscript. Wesley C. Kephart, Coree D. Pledge, Paul A. Roberson, Petey W. Mumford, Matthew A. Romero, Christopher B. Mobley, Jeffrey S. Martin, Kaelin C. Young, Ryan P. Lowery, Jacob M. Wilson, Kevin W. Huggins and Michael D. Roberts revised and critically edited the manuscript and agree to be accountable for all aspects of the work and ensuring that questions related to the accuracy or integrity of any part of the work are appropriately investigated and resolved.

Conflicts of Interest: Ryan P. Lowery and Jacob M. Wilson are actively involved in ketogenic salt patents, and have authored a for-profit book describing ketogenic diets (The Ketogenic Bible: An Authoritative Guide to Ketosis). None of the other authors have conflicts of interest in publishing these data. The only costs herein were the serum analyses, which were funded through residual funds provided to Michael D. Roberts through a prior grant awarded to him by the Applied Sports Performance Institute (ASPI). No salaries or royalties were received from ASPI for this project.

\section{References}

1. Longland, T.M.; Oikawa, S.Y.; Mitchell, C.J.; Devries, M.C.; Phillips, S.M. Higher compared with lower dietary protein during an energy deficit combined with intense exercise promotes greater lean mass gain and fat mass loss: A randomized trial. Am. J. Clin. Nutr. 2016, 103, 738-746. [CrossRef] [PubMed]

2. Antonio, J.; Peacock, C.A.; Ellerbroek, A.; Fromhoff, B.; Silver, T. The effects of consuming a high protein $\operatorname{diet}(4.4 \mathrm{~g} / \mathrm{kg} / \mathrm{d})$ on body composition in resistance-trained individuals. J. Int. Soc. Sports Nutr. 2014, 11, 19. [CrossRef] [PubMed]

3. Antonio, J.; Ellerbroek, A.; Silver, T.; Orris, S.; Scheiner, M.; Gonzalez, A.; Peacock, C.A. A high protein diet $(3.4 \mathrm{~g} / \mathrm{kg} / \mathrm{d})$ combined with a heavy resistance training program improves body composition in healthy trained men and women-A follow-up investigation. J. Int. Soc. Sports Nutr. 2015, 12, 39. [CrossRef] [PubMed]

4. Campbell, B.; Kreider, R.B.; Ziegenfuss, T.; La Bounty, P.; Roberts, M.; Burke, D.; Landis, J.; Lopez, H.; Antonio, J.; Taylor, L.W.; et al. International society of sports nutrition position stand: Protein and exercise. J. Int. Soc. Sports Nutr. 2007, 4, 8. [CrossRef] [PubMed]

5. Roberts, M.D.; Holland, A.M.; Kephart, W.C.; Mobley, C.B.; Mumford, P.W.; Lowery, R.P.; Fox, C.D.; McCloskey, A.E.; Shake, J.J.; Mesquita, P.; et al. A putative low-carbohydrate ketogenic diet elicits mild nutritional ketosis but does not impair the acute or chronic hypertrophic responses to resistance exercise in rodents. J. Appl. Physiol. 2015, 120, 1173-1185. [CrossRef] [PubMed]

6. Holland, A.M.; Kephart, W.C.; Mumford, P.W.; Mobley, C.B.; Lowery, R.P.; Shake, J.J.; Patel, R.K.; Healy, J.C.; McCullough, D.J.; Kluess, H.A.; et al. Effects of a ketogenic diet on adipose tissue, liver, and serum biomarkers in sedentary rats and rats that exercised via resisted voluntary wheel running. Am. J. Physiol. Regul. Integr. Comp. Physiol. 2016, 311, R337-R351. [CrossRef] [PubMed]

7. Wilson, J.M.; Lowery, R.P.; Roberts, M.D.; Sharp, M.H.; Joy, J.M.; Shields, K.A.; Partl, J.; Volek, J.S.; D'Agostino, D. The effects of ketogenic dieting on body composition, strength, power, and hormonal profiles in resistance training males. J. Strength Cond. Res. 2017. [CrossRef] [PubMed]

8. Nilsson, J.; Ericsson, M.; Joibari, M.M.; Anderson, F.; Carlsson, L.; Nilsson, S.K.; Sjodin, A.; Buren, J. A low-carbohydrate high-fat diet decreases lean mass and impairs cardiac function in pair-fed female C57BL/6J mice. Nutr. Metab. (Lond.) 2016, 13, 79. [CrossRef] [PubMed]

9. Kennedy, A.R.; Pissios, P.; Otu, H.; Roberson, R.; Xue, B.; Asakura, K.; Furukawa, N.; Marino, F.E.; Liu, F.F.; Kahn, B.B.; et al. A high-fat, ketogenic diet induces a unique metabolic state in mice. Am. J. Physiol. Endocrinol. Metab. 2007, 292, E1724-E1739. [CrossRef] [PubMed]

10. Paoli, A.; Grimaldi, K.; D’Agostino, D.; Cenci, L.; Moro, T.; Bianco, A.; Palma, A. Ketogenic diet does not affect strength performance in elite artistic gymnasts. J. Int. Soc. Sports Nutr. 2012, 9, 34. [CrossRef] [PubMed]

11. Gregory, R.M.; Hamdan, H.; Torisky, D.M.; Akers, J.D. A low-carbohydrate ketogenic diet combined with 6-weeks of crossfit training improves body composition and performance. Int. J. Sports Exer. Med. 2017, 3, 1-10. [CrossRef] 
12. Burke, L.M.; Ross, M.L.; Garvican-Lewis, L.A.; Welvaert, M.; Heikura, I.A.; Forbes, S.G.; Mirtschin, J.G.; Cato, L.E.; Strobel, N.; Sharma, A.P.; et al. Low carbohydrate, high fat diet impairs exercise economy and negates the performance benefit from intensified training in elite race walkers. J. Physiol. 2017, 595, 2785-2807. [CrossRef] [PubMed]

13. Kephart, W.C.; Wachs, T.D.; Mac Thompson, R.; Brooks Mobley, C.; Fox, C.D.; McDonald, J.R.; Ferguson, B.S.; Young, K.C.; Nie, B.; Martin, J.S.; et al. Ten weeks of branched-chain amino acid supplementation improves select performance and immunological variables in trained cyclists. Amino Acids 2016, 48, 779-789. [CrossRef] [PubMed]

14. Haun, C.T.; Mumford, P.W.; Roberson, P.A.; Romero, M.A.; Mobley, C.B.; Kephart, W.C.; Anderson, R.G.; Colquhoun, R.J.; Muddle, T.W.D.; Luera, M.J.; et al. Molecular, neuromuscular, and recovery responses to light versus heavy resistance exercise in young men. Physiol. Rep. 2017, 5. [CrossRef] [PubMed]

15. Mobley, C.B.; Haun, C.T.; Roberson, P.A.; Mumford, P.W.; Romero, M.A.; Kephart, W.C.; Anderson, R.G.; Vann, C.G.; Osburn, S.C.; Pledge, C.D.; et al. Effects of whey, soy or leucine supplementation with 12 weeks of resistance training on strength, body composition, and skeletal muscle and adipose tissue histological attributes in college-aged males. Nutrients 2017, 9, 972. [CrossRef] [PubMed]

16. Kephart, W.C.; Mumford, P.W.; McCloskey, A.E.; Holland, A.M.; Shake, J.J.; Mobley, C.B.; Jagodinsky, A.E.; Weimar, W.H.; Oliver, G.D.; Young, K.C.; et al. Post-exercise branched chain amino acid supplementation does not affect recovery markers following three consecutive high intensity resistance training bouts compared to carbohydrate supplementation. J. Int. Soc. Sports Nutr. 2016, 13, 30. [CrossRef] [PubMed]

17. Volek, J.S.; Phinney, S.D. The Art and Science of Low Carbohydrate Performance; Beyond Obesity LLC: Miami, FL, USA, 2010.

18. Volek, J.S.; Phinney, S.D.; Forsythe, C.E.; Quann, E.E.; Wood, R.J.; Puglisi, M.J.; Kraemer, W.J.; Bibus, D.M.; Fernandez, M.L.; Feinman, R.D. Carbohydrate restriction has a more favorable impact on the metabolic syndrome than a low fat diet. Lipids 2009, 44, 297-309. [CrossRef] [PubMed]

19. Noakes, M.; Foster, P.R.; Keogh, J.B.; James, A.P.; Mamo, J.C.; Clifton, P.M. Comparison of isocaloric very low carbohydrate/high saturated fat and high carbohydrate/low saturated fat diets on body composition and cardiovascular risk. Nutr. Metab. (Lond.) 2006, 3, 7. [CrossRef] [PubMed]

20. Brehm, B.J.; Seeley, R.J.; Daniels, S.R.; D'Alessio, D.A. A randomized trial comparing a very low carbohydrate diet and a calorie-restricted low fat diet on body weight and cardiovascular risk factors in healthy women. J. Clin. Endocrinol. Metab. 2003, 88, 1617-1623. [CrossRef] [PubMed]

21. Gomez-Arbelaez, D.; Bellido, D.; Castro, A.I.; Ordonez-Mayan, L.; Carreira, J.; Galban, C.; Martinez-Olmos, M.A.; Crujeiras, A.B.; Sajoux, I.; Casanueva, F.F. Body composition changes after very-low-calorie ketogenic diet in obesity evaluated by 3 standardized methods. J. Clin. Endocrinol. Metab. 2017, 102, 488-498. [CrossRef] [PubMed]

22. Williams, C.; Rollo, I. Carbohydrate nutrition and team sport performance. Sports Med. 2015, 45 (Suppl. S1), S13-S22. [CrossRef] [PubMed]

23. Volek, J.S.; Freidenreich, D.J.; Saenz, C.; Kunces, L.J.; Creighton, B.C.; Bartley, J.M.; Davitt, P.M.; Munoz, C.X.; Anderson, J.M.; Maresh, C.M.; et al. Metabolic characteristics of keto-adapted ultra-endurance runners. Metabolism 2016, 65, 100-110. [CrossRef] [PubMed]

24. Zajac, A.; Poprzecki, S.; Maszczyk, A.; Czuba, M.; Michalczyk, M.; Zydek, G. The effects of a ketogenic diet on exercise metabolism and physical performance in off-road cyclists. Nutrients 2014, 6, 2493-2508. [CrossRef] [PubMed]

25. McSwiney, F.T.; Wardrop, B.; Hyde, P.N.; Lafountain, R.A.; Volek, J.S.; Doyle, L. Keto-adaptation enhances exercise performance and body composition responses to training in endurance athletes. Metabolism 2017. [CrossRef] [PubMed]

(C) 2018 by the authors. Licensee MDPI, Basel, Switzerland. This article is an open access article distributed under the terms and conditions of the Creative Commons Attribution (CC BY) license (http:/ / creativecommons.org/licenses/by/4.0/). 